\title{
Positive outcome in a high-grade myxofibrosarcoma: a case report
}

\author{
Mihaela Olaru*, Cornelia Nitipir*** \\ *Clinic of Medical Oncology, "Elias" University Emergency Hospital, Bucharest \\ **"Carol Davila" University of Medicine and Pharmacy, Bucharest
}

Correspondence to: Mihaela Olaru, MD, Clinic of Medical Oncology, "Elias" University Emergency Hospital, Bucharest, 17 Marasti Street, Bucharest, Romania, Mobile Phone: +40726107 947, E-mail: mihao611@yahoo.com

\begin{abstract}
Myxofibrosarcoma or myxoid malignant fibrous histiocytoma is one of the most common sarcomas of the limb. It is usually treated multimodally. Most frequent sites of metastasis are the bone, lung and lymph nodes. The present paper is a case report of a 65-year-old male with myxofibrosarcoma of the fibularis longus muscle, for which he first underwent surgery - tumor resection with appropriate margins. The tumor was staged $\mathrm{pT} 2 \mathrm{~b}$ cNo cMo. Postoperative PET-CT revealed metabolically inactive pulmonary nodules. Two months after surgery, he underwent adjuvant radiotherapy, a total dose of $60 \mathrm{~Gy}$ and 6 courses of chemotherapy (doxorubicin and ifosfamide). Pulmonary nodules have been stationary on all subsequent imagistic studies. He is free of recurrence on long-term follow-up.

Keywords: limb sarcoma, radiotherapy, chemotherapy
\end{abstract}

\section{Introduction}

Myxofibrosarcoma, also known as myxoid malignant fibrous histiocytoma, is a malignant fibroblastic lesion with variable myxoid matrix, pleomorphic cells, and distinctive vascular pattern. It is considered one of the most common sarcomas, arising in the limbs, and rarely in head and neck areas, trunk, and retroperitoneum, in elderly patients. It presents as an enlarged, painless mass, with a high local recurrence. Myxofibrosarcoma's most commonly metastases are bone, lung, and lymph nodes. The treatment of choice is surgical excision. As local recurrence is high, radiation therapy and/ or chemotherapy might be needed [1].

We present the case of a 65-year-old Caucasian male, smoker, with no significant past medical history, who was admitted to the Orthopaedics Department at "Elias" University Emergency Hospital, Bucharest, for diffuse swelling in his right calf, on 1st of March 2015. Magnetic resonance imaging showed that a tumor was located in the fibularis longus muscle. No lymph node or distant metastasis was detected. After biopsy was performed, the lesion was classified as high-grade myxofibrosarcoma, pT2b cNo cMo. The multidisciplinary team decided to proceed with the surgical treatment. The aim of the oncology surgical team was to resect a 
tumor with appropriate margins. A tumor was removed with a complete size of the resected tissue of $20 / 10 / 9 \mathrm{~cm}$.

The specimen obtained during surgery showed similar features to the one in the biopsy. Macroscopically, an ovoid tumor of $9 / 6 / 5 \mathrm{~cm}$, which extended to the deep fascia involving the muscle tissues, with negative margins, was described. The tumor was characterized by pleomorphic spindle cells, hyperchromatic, and irregular nuclei, exhibiting a focal myxoid matrix with a prominent vascular pattern. The immunohistochemistry showed tumor cells were negative for SMA and desmin, $\mathrm{CD}_{5} 6 ; \mathrm{CD}_{34}$ and $\mathrm{S}-100$ protein were positive, more than $50 \%$ of the tumor cells displayed p53 protein, and the proliferation-related $\mathrm{Ki} 67$ antigen index of $70 \%$, confirming the histological finding of a myxofibrosarcoma.

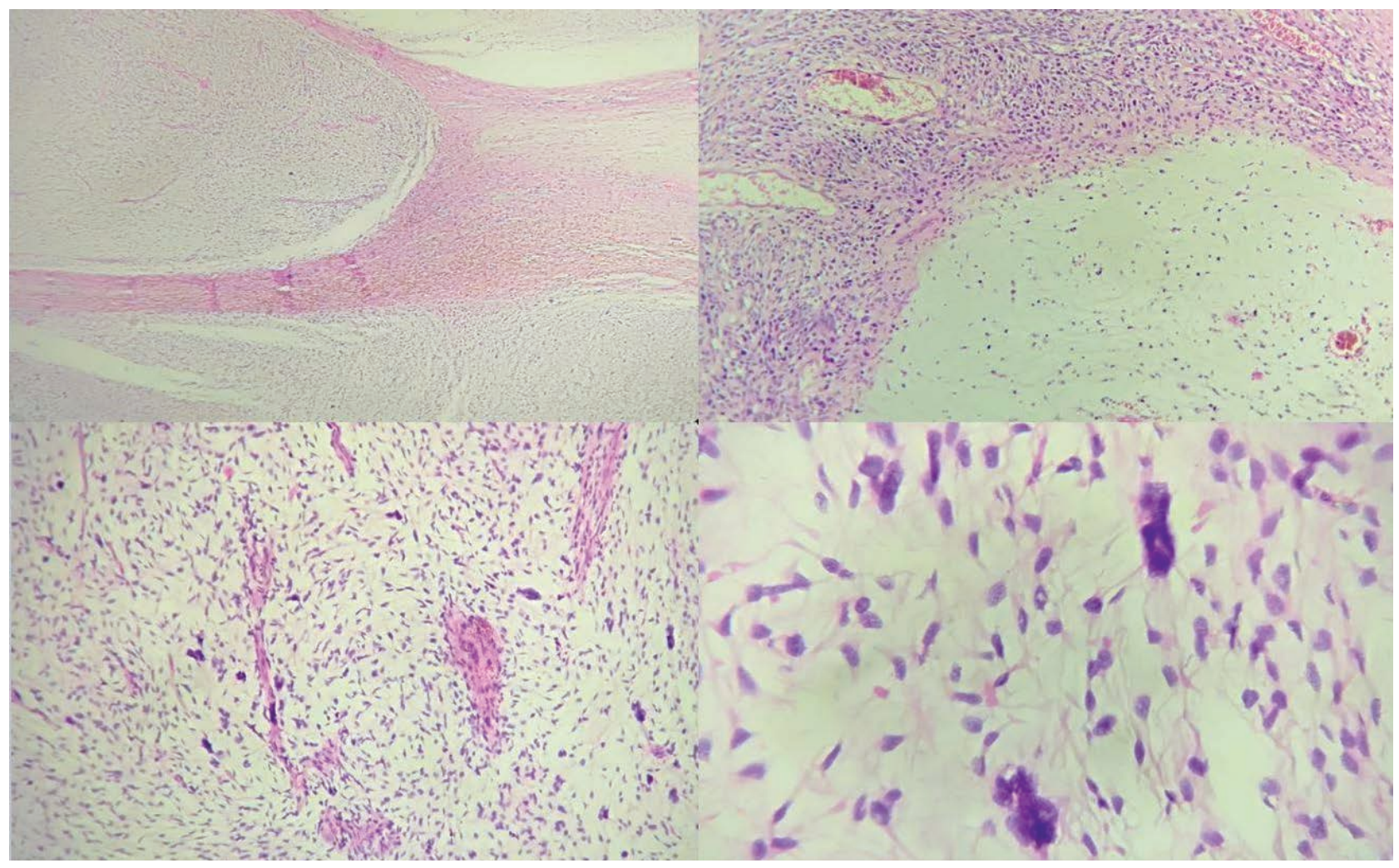

Fig. 1 Upper-Left corner: Multinodular tumor composed of pleomorphic spindle cells in the myxoid background (Hematoxylin and eosin; original magnification X40); Upper-Right corner: Spindle cells with large elongated hyperchromatic and irregular nuclei, transition from myxoid area to cellular area (Hematoxylin and eosin; original magnification X10); Lower-Left corner: Cellular proliferation with spindle cells and multinucleated giant cells (Hematoxylin and eosin; original magnification X20); Lower-Right corner: Large pleomorphic spindle cells distributed in a myxoid stroma (Hematoxylin and eosin; original magnification $X_{40}$ )

Positron Emission Tomography (PET) exam performed after 2 months showed a pulmonary nodule in the left inferior lobe with no metabolic activity. The patient was prior referred to an oncologist for further therapeutical conduct.

The initial recommended treatment was radiotherapy. It was decided to perform radiotherapy (25 sessions), with a total dose of $60 \mathrm{~Gy}$. The treatment was continued with 6 cycles of chemotherapy with doxorubicin d1-3 (20mg/ m2/ d) and ifosfamide d1-3 (2000mg/ $\mathrm{m} 2 /$ d) with mesna uroprotection at every three weeks. No degree of myelosuppression was observed during treatment, and no cardiotoxicity or other toxicities.

During his next three years of follow up, the patient was recurrence-free. All imaging results excluded any presence of suspect locoregional 
lymphadenopathies, with no local recurrence or distant metastasis.

\section{Discussion}

Myxofibrosarcoma, also known as myxoid malignant fibrous histiocytoma, is a malignant fibroblastic lesion that affects predominantly elderly patients, aged ranging from 60 to 80 years old and slight male predominance $[1,3]$. Myxofibrosarcomas often arise in lower and upper limbs (lower limb > upper limb) and rarely in head and neck areas, trunk, retroperitoneum, pelvis, hands and feet $[2,4]$. Unlike other sarcomas, myxofibrosarcomas are located subcutaneously and in deep muscular compartments. Approximately two-thirds are located in dermal or soft subcutaneous tissue, and one third are located in fascia and skeletal muscle $[2,4,5]$. Histopathologically, they are graded into high, intermediate, and low grade. The high-grade ones are marked by an intense cellularity defined by pleomorphic, atypical mitoses and spindled cells; and decreased myxoid matrix material $[2,4]$. The location and grade of malignancy act as predictors when it comes to myxofibrosarcoma prognostic. Regarding high-grade neoplasms, situated deep in the muscular compartments, the percentage of metastases and associated mortality is higher $[6,7]$. The high-grade myxofibrosarcoma can have metastases such as pulmonary, osseous and lymph nodes. Unrelated to the grade of malignancy, the local recurrence percentage ranges between 50 and $60 \%$, and when it does, the histological grade of the lesion is higher than the original one $[2,4,8]$.

Standard treatment in localized disease is surgery combined with radiation therapy. Adding adjuvant chemotherapy in high-grade soft-tissue sarcomas, to improve survival rates, is still a subject of controversy [9].

According to Enneking et al. (1980), a system for surgical staging is needed to differentiate between intralesional, marginal, wide, and radical resections [10].

Lately, amputation has been overthrown by limb-sparing when deciding surgical treatment in patients with soft tissue sarcoma. However, amputation becomes an option if wide surgical tumor is not possible, as the excision could lead to severe functional impairment, due to either the tumour's fixation to or infiltration of nerves, vessels, or bone [11].

In the current case, our patient had a radical surgery involving the resection of the entire compartment containing the tumor.

Another important aspect is surgical margin width. It is difficult to precisely define an optimal margin, considering both reducing local recurrence and preventing radical resection. According to UICC classification Ro, resection is defined by the coverage of the tumor of at least $1 \mathrm{~mm}$ of healthy tissue, whereas the R-classification defines Ro as tumor freemargins regardless of thickness [11].

For patients with soft tissue sarcoma, radiotherapy is added in selected cases and can be used either intra-operative or post-operative as adjuvant treatment, and can also be used in patients with inoperable tumors and/ or distant metastases as palliative treatment. For highgrade lesions (deeper than $5 \mathrm{~cm}$ ), standard treatment post surgery is radiation therapy [12].

In ourpatients' case, after several discussions between the multidisciplinary teams, taking into consideration all his prognostic factors such as age, tumor size and surgical margins and also histology and grade, a total dose of $60 G y$ of radiation therapy was decided.

In advanced disease with resectable pulmonary metastases, surgery is considered standard as long as the procedure is feasible and no other extrapulmonary metastases exist. PET-CT or abdominal CT and bone scan are in order to confirm that pulmonary metastases are isolated [12].

First-line treatment in patients with advanced disease is anthracyclines. Though there is no formal evidence that multiagent 
chemotherapy is superior to single-agent chemotherapy with doxorubicin alone, in terms of overall survival (OS), some studies have shown higher response rates in a number of high-grade soft-tissue sarcoma [13,14]. Taking into consideration all his prognostic factors, we decided for 6 cycles of AIM (doxorubicin, ifosfamide, mesna) q3w.

A multidisciplinary team is needed when treating myxofibrosarcoma, to overcome the challenges. Further studies are needed to intensify local treatment and establish the benefit of chemotherapy.

\section{References}

1. DeVita VT, Lawrence TS, Rosenberg SA. DeVita, Hellman, and Rosenberg's cancer: Principles \& practice of oncology. 10th ed., 2014, United States: Lippincott Williams and Wilkins, 1540.

2. Mentzel T, van den Berg E, Molenaar W. Myxofibrosarcoma. In: Fletcher C, Unni K, Mertens F. WHO classification of tumors - pathology and genetics, tumors of soft tissue and bone. 2002, Lyon: IARC Press, 102-1031.

3. Castro BAC, Piancastelli ACC, Meyer RLB, Piancastelli PM, Ribeiro CA, Miranda RMC. Myxofibrosarcoma - Case report. 2016; 91(1):97-9.

4. Dodd LG, Bui MM. Atlas of Soft Tissue and Bone Pathology: With Histologic, Cytologic, and Radiologic Correlations. 2015, New York: Demos Medical, 68-71.

5. Schepper AM, Vanhoenacker F, Gielen J, Parizel PM. Imaging of soft tissue tumors. 3. 2006, Berlin Heidelberg New York: Springer-Verlag, 196-198.

6. Mentzel T, Calonje E, Wadden C, Camplejohn RS, Beham A, Smith MA, Fletcher CD. Myxofibrosarcoma. Clinicopathologic analysis of 75 cases with emphasis on the low-grade variant. Am J SurgPathol. 1996; 20:391-405.

7. Merck C, Angervall L, Kindblom LG, Oden A. Myxofibrosarcoma. A malignant soft tissue tumor of fibroblastichistiocytic origin. A clinicopathologic and prognostic study of 110 cases using multivariate analysis. Acta PatholMicrobiol Immunol Scand. 1953; Suppl 282:1-40.

8. Weiss SW, Enzinger FM. Myxoid variant of malignant fibrous histiocytoma. Cancer. 1977; 39:1672-1685.

9. Adjuvant chemotherapy for localised resectable soft-tissue sarcoma of adults: meta-analysis of individual data. Sarcoma Meta-analysis Collaboration. Lancet. 1997; 350:1647-54.

10. Enneking WF, Spanier SS, Goodman MA. A system for the surgical staging of musculoskeletal sarcoma. ClinOrthopRelat Res. 1980; 153:106-120.

11. Smolle MA, Dimosthenis A, Per-Ulf T, Szkandera J, LieglAtzwanger B, Leithner A. Diagnosis and treatment of soft-tissue sarcomas of the extremities and trunk. EFORT Open Rev. 2017; 2:421-431.

12. The ESMO/European Sarcoma Network Working Group. Soft tissue and visceral sarcomas: ESMO Clinical Practice Guidelines for diagnosis, treatment, and follow-up. Ann Oncol. 2014; 25:iii102-iii112.

13. Judson I, Verweij J, Gelderblom H et al. European Organisation and Treatment of Cancer Soft Tissue and Bone Sarcoma GroupDoxorubicin alone versus intensified doxorubicin plus ifosfamide for first-line treatment of advanced or metastatic soft-tissue sarcoma: a randomised controlled phase 3 trial. Lancet Oncol. 2014; 15:415-423.

14. Antman K, Crowley J, Balcerzak SP et al. An intergroup phase III randomized study of doxorubicin and dacarbazine with or without ifosfamide and mesna in advanced soft tissue and bone sarcomas. J ClinOncol. 1993; 11:1276-1285. 